\title{
Fractional Brownian motion approach to polymer translocation: The governing equation of motion
}

\author{
J. L. A. Dubbeldam, ${ }^{2}$ V. G. Rostiashvili, ${ }^{1}$ A. Milchev,${ }^{1,3}$ and T. A. Vilgis ${ }^{1}$ \\ ${ }^{1}$ Max Planck Institute for Polymer Research, 10 Ackermannweg, D-55128 Mainz, Germany \\ ${ }^{2}$ Delft University of Technology, NL-2628CD Delft, The Netherlands \\ ${ }^{3}$ Institute for Physical Chemistry, Bulgarian Academy of Sciences, BG-1113 Sofia, Bulgaria
}

(Received 22 July 2010; published 12 January 2011)

\begin{abstract}
We suggest a governing equation that describes the process of polymer-chain translocation through a narrow pore and reconciles the seemingly contradictory features of such dynamics: (i) a Gaussian probability distribution of the translocated number of polymer segments at time $t$ after the process has begun, and (ii) a subdiffusive increase of the distribution variance $\Delta(t)$ with elapsed time $\Delta(t) \propto t^{\alpha}$. The latter quantity measures the meansquared number $s$ of polymer segments that have passed through the pore $\Delta(t)=\left\langle[s(t)-s(t=0)]^{2}\right\rangle$, and is known to grow with an anomalous diffusion exponent $\alpha<1$. Our main assumption [i.e., a Gaussian distribution of the translocation velocity $v(t)]$ and some important theoretical results, derived recently, are shown to be supported by extensive Brownian dynamics simulation, which we performed in 3D. We also numerically confirm the predictions made recently that the exponent $\alpha$ changes from 0.91 to 0.55 to 0.91 for short-, intermediate-, and long-time regimes, respectively.
\end{abstract}

DOI: 10.1103/PhysRevE.83.011802

PACS number(s): 82.35.Lr, 87.15.A-, 87.16.dp

\section{INTRODUCTION}

Polymer translocation has recently attracted a good amount of attention as it plays a crucial role in processes such as DNA and RNA transport through membrane channels [1]. Ultimately, this research might lead to a longstanding objective of nucleotic transport: fast and cheap sequencing of DNA. Meanwhile, many interesting experimental and theoretical findings have been reported [2]. A number of important observations have been gained by means of computer simulations [3-9]. However, notwithstanding the significance of translocation phenomena both as a possible technological application and from the standpoint of basic research, the understanding of the polymer translocation through a narrow pore is still elusive and, in some respects, controversial [10].

In most theoretical descriptions (except Ref. [10], where a full Cartesian-space treatment has been suggested), the translocation process is assumed to be captured by a single variable, the translocation coordinate $s(t)$, which measures the number of translocated segments at time $t$. Generally, $s(t)$ would depend on the external potential, if present, and be influenced by random noise. At present, it is well established that the translocation coordinate $s(t)$ follows an anomalous, subdiffusive law, although the physical background as well as the equation that governs the probability distribution function (PDF) $W(s, t)$ that $s$ segments have passed through the pore at time $t$ are still controversial.

It was suggested recently [11] that the translocation dynamics is governed by a fractional diffusion equation (FDE) with an (anomalous) diffusion exponent $\alpha<1$. Thus, a description of the translocation process in terms of $W(s, t)$ was derived [5], which appeared to agree favorably with Monte Carlo simulation results. This approach was generalized [6] to the case of biased translocation, driven by external potential, which was described by an appropriate fractional Fokker-Planck equation (FFPE). As for the nondriven translocation, however, the FFPE approach yields a non-Gaussian distribution $W(s, t)$, the width $\Delta(t)$ of which diverges subdiffusionally with the elapsed time $\Delta(t) \propto t^{\alpha}$. While this latter property may readily be verified in numeric experiments, the unambiguous determination of the precise functional shape of $W(s, t)$ is rather difficult as a result of progressively deteriorating statistics of the distribution at late times.

Meanwhile, several recent publications [12-14], devoted to the unbiased translocation dynamics of a Gaussian onedimensional (1D) chain [12] as well as to that of a twodimensional (2D) self-avoiding chain [13], have validated the subdiffusive behavior of $\left\langle s^{2}(t)\right\rangle$ with an exponent $\alpha \simeq 0.8$. Nonetheless, these new findings cast serious doubts as to whether the FFPE indeed provides an adequate description of nondriven translocation dynamics:

(i) It was found [12-14] by means of computer simulations that the probability distribution $W(s, t)$ of the translocation coordinate $s$ stays Gaussian for different time moments not exceeding the mean translocation time $\langle\tau\rangle$. At larger times, this distribution attains a more complex form.

(ii) The long-time tail of the first-passage-time-distribution (FPTD) was found to be exponentially decreasing, thus challenging the power-law behavior, suggested earlier [5] within the framework of the FFPE.

These findings question the validity of the FDE approach in the description of translocation dynamics. Indeed, one can not reconcile the aforementioned controversial features of the translocation dynamics within a FFPE description.

In this work, we revisit the problem and demonstrate that a proper Fokker-Planck equation of motion (FPEM), which governs the PDF $W(s, t)$ and faithfully reproduces all recently found properties of translocation dynamics, may be rationalized and solved within the framework of fractional Brownian motion (fBm) [15]. Namely, we treat the definition of the translocation coordinate velocity $v(t)=d s(t) / d t$ as a Langevin equation, where the velocity auto-correlation function $G(t)=\langle v(t) v(0)\rangle$ is considered as dynamic input without making any a priori assumption about the dynamics that underlies the translocation process. In principle, $G(t)$ may be taken from the computer simulation. Such an approach finally leads to a FPEM (with a time-dependent diffusion 
coefficient) for $W(s, t)$ in unbiased and biased cases. We make an extensive Brownian dynamics simulation study (using the GROMACS simulation package) in order to check and justify our analytical results.

In Sec. II, we derive a governing equation for $\operatorname{PDF} W(s, t)$, calculate its first moments, and present an exact analytic solution for the first-passage-time distribution of translocation times as well as for the so-called survival probability in terms of monomer consecutive number $s$ and elapsed time $t$. A comparison with our Brownian dynamics simulation data is performed in Sec. III. Our main conclusions are then given as a brief summary in Sec. IV.

\section{CHAIN TRANSLOCATION DYNAMICS}

\section{A. Fokker-Planck equation with a time-dependent drift and diffusion coefficient}

One may derive the FPEM for the distribution $W(s, t)$, starting from the Langevin equation

$$
\frac{d}{d t} s(t)=v(t)
$$

where, by assumption, the translocation velocity $v(t)=$ $d s(t) / d t$ follows Gaussian statistics (i.e., it is MaxwellBoltzmann distributed). If a (generally time-dependent) external driving force $f(t)$ is present, the mean velocity $\langle v(t)\rangle=$ $f(t) / \xi_{0}$, where $\xi_{0}$ denotes the friction coefficient. Note that the velocity $v(t)$ in Eq. (1) reflects the change in the $s$ coordinate per unit time. By measuring $s(t)$ as the contour length of the chain on the trans-side of the membrane, a reasonable estimate for the translocation velocity is obtained. In fact, $v(t)$ can be approximated by the velocity of the bead inside the pore in the direction perpendicular to the wall, which we denote by $v_{z}(t)$ (see Fig. 1). Thus, we tacitly assume that translocation velocity may be faithfully characterized by bead velocity in Cartesian space. The correctness of this approximation will be addressed in Sec. III

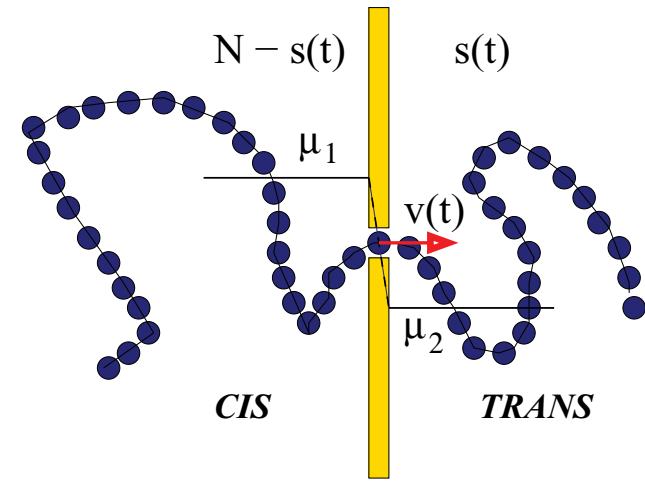

FIG. 1. (Color online) Chain translocation through a nanopore with an instantaneous translocation coordinate $s(t)$. The translocation velocity is approximated by the velocity of the bead that resides inside the pore. The separating membrane is taken as sufficiently thin so that no more than one bead may be in the pore at a time. The driving force caused by the chemical potential gradient within the pore $f=\left(\mu_{1}-\mu_{2}\right) / T$ acts on the same bead. The $\mu_{1}$ and $\mu_{2}$ denote values of the chemical potential on both sides of the separating membrane.
The variance of $s(t)$ can easily be calculated under the assumption that the velocity auto-correlation function $\left\langle v\left(t_{1}\right) v\left(t_{2}\right)\right\rangle$, defined as $\left\langle v\left(t_{1}\right) v\left(t_{2}\right)\right\rangle=\lim _{T \rightarrow \infty}(1 / T) \int_{0}^{T}$ $v\left(t_{1}+t\right) v\left(t_{2}+t\right) d t$, depends on the difference $\left|t_{1}-t_{2}\right|$ only (assuming time translational invariance). This leads to the relationship [16]

$$
\left\langle\left(s-s_{0}\right)^{2}(t)\right\rangle=2 \int_{0}^{t}\left(t-t^{\prime}\right)\left\langle v\left(t^{\prime}\right) v(0)\right\rangle d t^{\prime},
$$

where $s_{0} \equiv s(t=0)$ is the initial value of the translocation coordinate. In the case of biased translocation, the driving force is usually caused by a chemical potential gradient $\Delta \mu$, which is typically generated by applying a voltage difference across the membrane, i.e., $f=\Delta \mu / T$, where $\Delta \mu=\mu_{1}-\mu_{2}$. Here, $f$ is imposed on the monomer that is currently in the pore (cf. Fig. 1). As pointed out above, the velocity $v(t)$ is assumed to be a Gaussian random variable with the first two cumulants given as

$$
\langle v(t)\rangle=\frac{f(t)}{\xi_{0}}
$$

and

$$
G\left(t_{1}, t_{2}\right) \equiv\left\langle\left[v\left(t_{1}\right)-\left\langle v\left(t_{1}\right)\right\rangle\right]\left[v\left(t_{2}\right)-\left\langle v\left(t_{2}\right)\right\rangle\right]\right\rangle .
$$

We next consider the PDF $W(s, t)$, which is defined as

$$
W(s, t)=\left\langle\delta\left(s-s_{0}-\int_{0}^{t} v\left(t^{\prime}\right) d t^{\prime}\right)\right\rangle,
$$

and satisfies the initial condition $W(s, 0)=\delta\left(s-s_{0}\right)$. It is straightforward to show that

$$
\begin{aligned}
\frac{\partial}{\partial t} W(s, t)= & -\frac{\partial}{\partial s}\langle v(t)\rangle\left[\left\langle\delta\left(s-s_{0}-\int_{0}^{t} v\left(t^{\prime}\right) d t^{\prime}\right)\right\rangle\right] \\
& -\frac{\partial}{\partial s} \int_{0}^{t} d \tau G(t, \tau)\left\langle\frac { \delta } { \delta v ( \tau ) } \delta \left( s-s_{0}\right.\right. \\
& \left.\left.-\int_{0}^{t} v\left(t^{\prime}\right) d t^{\prime}\right)\right\rangle,
\end{aligned}
$$

where we have used Novikov's theorem [17,18] with the two first cumulants of $v(t)$ given by Eqs. (3) and (4). In the last term in Eq. (6), we have also used $\langle[\delta / \delta v(\tau)] \delta(s-$ $\left.\left.s_{0}-\int_{0}^{t} v\left(t^{\prime}\right) d t^{\prime}\right)\right\rangle=-(\partial / \partial s)\left\langle\delta\left(s-s_{0}-\int_{0}^{t} v\left(t^{\prime}\right) d t^{\prime}\right)\right\rangle$ as well as $\int_{0}^{t} \delta\left(\tau-t^{\prime}\right) d t^{\prime}=1$ for $\tau \leqslant t$. Eventually, the FPEM takes on the form

$$
\frac{\partial}{\partial t} W(s, t)=-\frac{f(t)}{\xi_{0}} \frac{\partial}{\partial s} W(s, t)+\mathcal{D}(t) \frac{\partial^{2}}{\partial s^{2}} W(s, t),
$$

with a time-dependent diffusion coefficient $\mathcal{D}(t)=$ $\int_{0}^{t} G(t, \tau) d \tau$. This equation of motion is different from the FFPE [19], which was used in our previous investigation $[5,6]$. In contrast to the FFPE, it is Markovian but contains a time-dependent drift term and a time-dependent diffusion coefficient. Moreover, the form of Eq. (7) does not depend on the underlying dynamics (underdamped or overdamped regimes!) as far as the driving force $f(t)$ and the velocity correlator $G\left(t_{1}, t_{2}\right)$ are not specified. We should emphasize that Eq. (7) has been derived by assuming that the effective friction coefficient $\xi_{0}$ remains constant with time. This is supported 
by computer simulation data (cf. Fig. 6a in Bhattachrya et al. [20]). It was shown [20] that, for a driven translocation, the mean center-of-mass velocity quickly saturates to a constant value after a short initial increase. The transient regime itself lasts less than $10 \%$ of the total translocation time for a short chain $(N=128)$ and quickly vanishes for longer chains. Recently, the translocation dynamics has been treated [21,22] on the basis of a FPEM with a time-dependent drift (even for a constant driving force) and diffusion coefficient.

In the simplest case of an unbiased process (i.e., $f=0$ ), the system is in a steady state and $\mathcal{D}(t)=\int_{0}^{t} G(t-\tau) d \tau=$ $\int_{0}^{t} G(\tau) d \tau$. Then, with the boundary conditions at infinity, Eq. (7) may be solved by defining a new time variable $\theta$ :

$$
\frac{d \theta}{d t}=\int_{0}^{t} d t^{\prime} G\left(t^{\prime}\right)
$$

i.e., $\theta(t)=\int_{0}^{t} d t^{\prime} \int_{0}^{t^{\prime}} d t^{\prime \prime} G\left(t^{\prime \prime}\right)=\int_{0}^{t}\left(t-t^{\prime}\right) G\left(t^{\prime}\right) d t^{\prime}$. A comparison with Eq. (2) then yields

$$
\theta(t)=\frac{1}{2}\left\langle\left(s-s_{0}\right)^{2}\right\rangle \propto t^{\alpha}
$$

where we have assumed that the subdiffusive behavior of $\langle(s-$ $\left.\left.s_{0}\right)^{2}(t)\right\rangle$ is described by an exponent $\alpha<1$. Thus, Eq. (7) takes on the form

$$
\frac{\partial}{\partial \theta} \tilde{W}(s, \theta)=\frac{\partial^{2}}{\partial s^{2}} \tilde{W}(s, \theta) .
$$

The solution of Eq. (10) reads as $\tilde{W}(s, \theta)=(1 / 2 \pi \theta)^{1 / 2}$ $\exp \left[-\left(s-s_{0}\right)^{2} / 4 \theta\right]$. Then, after taking into account Eq. (9), one finds

$$
W(s, t)=\frac{1}{\sqrt{2 \pi D t^{\alpha}}} \exp \left[-\frac{\left(s-s_{0}\right)^{2}}{4 D t^{\alpha}}\right],
$$

where $D$ is a constant. This result for $W(s, t)$ has been obtained very recently by Panja $[23,24]$ in a more general context dedicated to anomalous polymer dynamics.

Evidently, the distribution $W(s, t)$ is indeed Gaussian, albeit with a width proportional to the second moment that grows subdiffusively with elapsed time. This result reproduces the Monte Carlo simulation findings, reported recently by Kantor and Kardar [12,13]. It should be noted that such a process, described by a Gaussian distribution with anomalous width, is sometimes referred to as fractional Brownian motion $[15,25]$ and has been discussed in the context of DNA sequence statistics [26].

\section{B. Boundary conditions}

Equation (10) should be considered along with the appropriate boundary conditions. In the special case when the translocation starts from the middle monomer (i.e., $s_{0}=N / 2$ ) and stops as soon as any of the two chain ends pass the pore, both boundary conditions are adsorbing, i.e., $\tilde{W}(s=0, \theta)=0$ and $\tilde{W}(s=N, \theta)=0$. The corresponding initial condition reads $\tilde{W}(s, \theta=0)=\delta\left(s-s_{0}\right)$. Recall that $\theta(t=0)=0$.

The full solution can be represented as a sum over eigenfunctions $\varphi_{n}(s)$, i.e., $\tilde{W}(s, \theta)=\sum_{n=0}^{\infty} T_{n}(\theta) \varphi_{n}(s)$ (see, e.g., [27]), where $\varphi_{n}(s)$ obey the equations $\left(d^{2} / d s^{2}\right) \varphi_{n}(s)+$ $\lambda_{n} \varphi_{n}(s)=0$. The temporal part is governed by the equation $(d / d \theta) T_{n}(\theta)+\lambda_{n} T_{n}(\theta)=0$ with eigenvalues $\lambda_{n}$. The proper eigenfunctions that satisfy both boundary conditions are $\varphi_{n}(s)=A_{n} \sin (n \pi / N)$ (the factor $A_{n}$ can be fixed by the initial condition). The eigenvalues are then $\lambda_{n}=$ $(\pi n / N)^{2}$. The completeness relation for the eigenfunctions (see Sec. 5.4 in [27]) has, in this case, the form $\delta(s-$ $\left.s_{0}\right)=\frac{2}{N} \sum_{n=0}^{\infty} \sin \left(\frac{n \pi s}{N}\right) \sin \left(\frac{n \pi s_{0}}{N}\right)$, so that the full solution thus becomes

$\tilde{W}(s, \theta)=\frac{2}{N} \sum_{n=0}^{\infty} \sin \left(\frac{n \pi s}{N}\right) \sin \left(\frac{n \pi s_{0}}{N}\right) \exp \left[-\left(\frac{n \pi}{N}\right)^{2} \theta\right]$.

Note that Eq. (9) defines a subdiffusional law $\theta(t)=D t^{\alpha}$, which governs the solution Eq. (12). Eventually, if the starting point $s_{0}=N / 2$ [as is the case in our molecular dynamics (MD) simulation], only the odd terms in the series [Eq. (12)] survive, i.e., $n=2 m+1$ and the final result reads as

$$
\begin{aligned}
W(s, t)= & \frac{2}{N} \sum_{m=0}^{\infty}(-1)^{m} \sin \left[\frac{(2 m+1) \pi s}{N}\right] \\
& \times \exp \left[-\frac{(2 m+1)^{2} \pi^{2}}{N^{2}} D t^{\alpha}\right] .
\end{aligned}
$$

The first two moments generated by this probability distribution contain a lot of information, which permits us to compare our theoretical results with those from literature and with the numerical results of Sec. III.

\section{First and second moments of $W(s, t)$}

Taking into account that $\int_{0}^{N} \sin [(2 m+1) \pi s / N] d s=$ $2 N /(2 m+1) \pi$ and $\int_{0}^{N} s \sin [(2 m+1) \pi s / N] d s=N^{2} /$ $(2 m+1) \pi$, one obtains for the first moment $\langle s\rangle=\int_{0}^{N} s W$ $(s, t) d s / \int_{0}^{N} W(s, t) d s=s_{0}=N / 2$. The second moment is centered at $s_{0}$, hence, $\Delta \equiv\left\langle s^{2}\right\rangle-\langle s\rangle^{2}=\int_{0}^{N}\left(s-s_{0}\right)^{2}$ $W(s, t) d s / \int_{0}^{N} W(s, t) d s$. This leads to the following expression for the variance:

$$
\begin{aligned}
\Delta & \equiv\left\langle s^{2}\right\rangle-\langle s\rangle^{2} \\
& =\frac{N^{2}}{4}\left\{1-\frac{8 \sum_{m=0}^{\infty} \frac{(-1)^{m}}{(2 m+1)^{3}} \exp \left[-\frac{(2 m+1)^{2} \pi^{2}}{N^{2}} D t^{\alpha}\right]}{\pi^{2} \sum_{m=0}^{\infty} \frac{(-1)^{m}}{(2 m+1)} \exp \left[-\frac{(2 m+1)^{2} \pi^{2}}{N^{2}} D t^{\alpha}\right]}\right\} .
\end{aligned}
$$

At $t \rightarrow \infty$, the second moment $\Delta$ reaches a plateau, i.e., $\Delta=\left(N^{2} / 4\right)\left(1-8 / \pi^{2}\right)$. It can be readily shown [taking into account that $\sum_{m=0}^{\infty}(-1)^{m} /(2 m+1)=\pi / 4$ and $\left.\sum_{m=0}^{\infty}(-1)^{m} /(2 m+1)^{3}=\pi^{3} / 32\right]$ that $\Delta(t=0)=0$. The time dependence of the second moment $\Delta$, given by Eq. (14), will be discussed further in Sec. III. 


\section{First-passage-time distribution}

The FPTD function is defined as in [27] and describes the probability distribution of the observed translocation times:

$$
Q(t)=-\frac{d}{d t} \int_{0}^{N} W(s, t) d s .
$$

Taking into account Eq. (13), this definition yields

$$
\begin{aligned}
Q(t)= & \frac{4 \pi \alpha D t^{\alpha-1}}{N^{2}} \sum_{m=0}^{\infty}(-1)^{m}(2 m+1) \\
& \times \exp \left[-\frac{(2 m+1)^{2} \pi^{2}}{N^{2}} D t^{\alpha}\right] .
\end{aligned}
$$

The long-time behavior of $Q$ is determined by the smallest eigenvalue in the series [Eq. (16)]. Therefore, at $t \rightarrow \infty$, one has

$$
Q(t)=\frac{4 \pi \alpha D t^{\alpha-1}}{N^{2}} \exp \left(-\frac{\pi^{2}}{N^{2}} D t^{\alpha}\right),
$$

i.e., the FPTD follows a stretched-exponential law at late times. We will try to check this prediction in our MD simulation study in Sec. III.

\section{E. Asymptotic behavior near the adsorbing boundary}

An interesting aspect of translocation dynamics in a system with two adsorbing boundaries has been considered recently [12-14], suggesting that, for a sufficiently long-time interval, the normalized distribution

$$
p_{s_{0}}(s, t)=\frac{W\left(s, s_{0}, t\right)}{\int_{0}^{N} W\left(s, s_{0}, t\right) d s}
$$

reaches a stable (time-independent) shape, which differs from the simple sine function, and at $s \rightarrow 0$ (i.e., close to the adsorbing boundary), the function $p_{s_{0}}(s) \sim s^{\phi}$, where $\phi>1$ (for a sine function, it would have been $\phi=1$ ).

Based on numerical results [12,13], one has tried [28] recently to link translocation dynamics to self-affine processes undergoing anomalous diffusion in bounded domains within the context of fBm. The argumentation [28] relies on two crucial assumptions: (i) The $\operatorname{PDF} W\left(s, s_{0}, t\right)$ has a self-affine form $W\left(s, s_{0}, t\right)=\left(1 / t^{\alpha / 2}\right) F\left(s / t^{\alpha / 2}, s_{0} / t^{\alpha / 2}\right)$, and (ii) the socalled survival probability $S\left(s_{0}, t\right)=\int_{0}^{N} W\left(s, s_{0}, t\right) d s$ has a long-time scaling behavior $S\left(s_{0}, t\right) \sim t^{-\theta}$ with an exponent $\theta=1-\alpha / 2$. Simple scaling arguments lead Zoia et al. [28] to the conclusion that the normalized probability $p_{s_{0}}(s, t)=$ $\left(1 / t^{\alpha / 2}\right) \tilde{p}_{y_{0}}(y)$, where $y=s / t^{\alpha / 2}, y_{0}=s_{0} / t^{\alpha / 2}$, and the scaling function $\tilde{p}_{y_{0}}(y) \sim y^{\phi}$, with the exponent $\phi=2 / \alpha-$ $1 \geqslant 1$.

Note that our solution, Eq. (12), disagrees with the scaling function proposed in [28]. Since the eigenvalue spectrum is discrete (as it should be for a finite interval $0 \leqslant s \leqslant N$ ), only the smallest eigenvalue dominates in the long-time limit, so for the PDF $W\left(s, s_{0}, t\right)$ and the survival probability $S\left(s_{0}, t\right)$ we get

$$
\begin{aligned}
W\left(s, s_{0}, t\right) & \sim\left(\frac{2}{N}\right) \sin \left(\frac{\pi s_{0}}{N}\right) \sin \left(\frac{\pi s}{N}\right) \exp \left[-\left(\frac{\pi}{N}\right)^{2} D t^{\alpha}\right], \\
S\left(s_{0}, t\right) & \sim\left(\frac{4}{\pi}\right) \sin \left(\frac{\pi s_{0}}{N}\right) \exp \left[-\left(\frac{\pi}{N}\right)^{2} D t^{\alpha}\right] .
\end{aligned}
$$

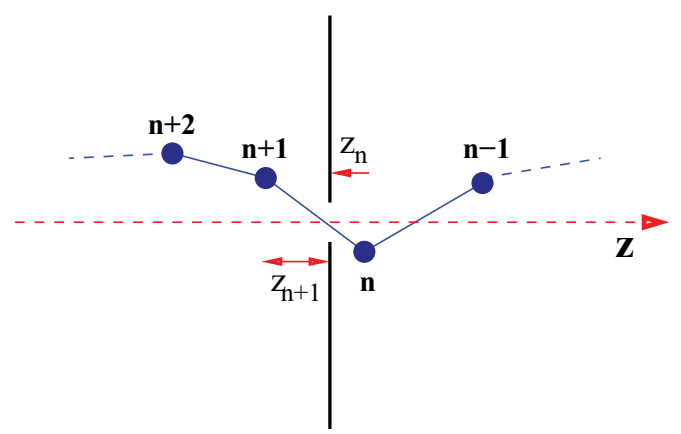

FIG. 2. (Color online) The individual chain segments are enumerated starting from the rightmost terminal bead. The origin of the coordinate system is placed in the pore. The $z$ coordinates of $n$ and $n+1$ beads are marked as $z_{n}$ and $z_{n+1}$, respectively.

Therefore,

$$
p_{s_{0}}(s, t)=\frac{W\left(s, s_{0}, t\right)}{S\left(s_{0}, t\right)}=\frac{\pi}{2 N} \sin \left(\frac{\pi s}{N}\right) .
$$

Evidently, the self-affine scaling [28], conjectured for $W\left(s, s_{0}, t\right)$ and $S\left(s_{0}, t\right)$, does not hold and the "stable shape" at $t \rightarrow \infty$ is a simple sine function. This behavior is in accordance with the definition of $\mathrm{fBm}$ [15]. The stretched-exponential behavior for the survival probability, which corresponds to Eq. (19), has been recently discussed in Ref. [29].

\section{SIMULATION RESULTS}

To study the translocation process numerically, we performed Brownian dynamics (BD) simulations for two different chain lengths: $N=51$ and $N=101$. The polymer was modeled using a coarse-grained description in which the adjacent monomers are connected by finitely extensible nonlinear elastic (FENE) springs, corresponding to a pair potential

$$
U_{\mathrm{FENE}}\left(r_{i j}\right)=-\frac{k r_{i j}^{2}}{2} \ln \left(1-\frac{r_{i j}^{2}}{R_{0}^{2}}\right),
$$

where $r_{i j}$ is the bond length between two beads and $R_{0}=1.5$ denotes its maximal extension. All beads experience excluded volume interactions, which are modeled by a Lennard-Jones potential $U_{\mathrm{LJ}}$, defined by

$$
U_{\mathrm{LJ}}\left(r_{i j}\right)=4 \epsilon\left[\left(\frac{\sigma}{r_{i j}}\right)^{12}-\left(\frac{\sigma}{r_{i j}}\right)^{6}\right],
$$

where we use a cutoff $r_{c}=2^{-1 / 6} \sigma$, implying that $U_{\mathrm{LJ}}=0$ for $r_{i j}>r_{c}$. The parameter values were taken as $\epsilon=1.0, \sigma=1.0$, and $k=30.0$, and were kept fixed during the simulations. The friction parameter $\xi$ was taken as $\xi=100 \sqrt{m \epsilon} / \sigma$, and the temperature $T=1.2 \epsilon / k_{\mathrm{B}}$, and this implies a monomer diffusion coefficient $D_{0}=k_{\mathrm{B}} T / \xi=1.2 \epsilon / \xi$.

In order to simulate a translocation event, we create a separating membrane, which consists of a monoatomic layer with a hole (the center monomer was removed). The membrane is placed inside a box of size $(54 \times 54 \times 54)$ with periodic 


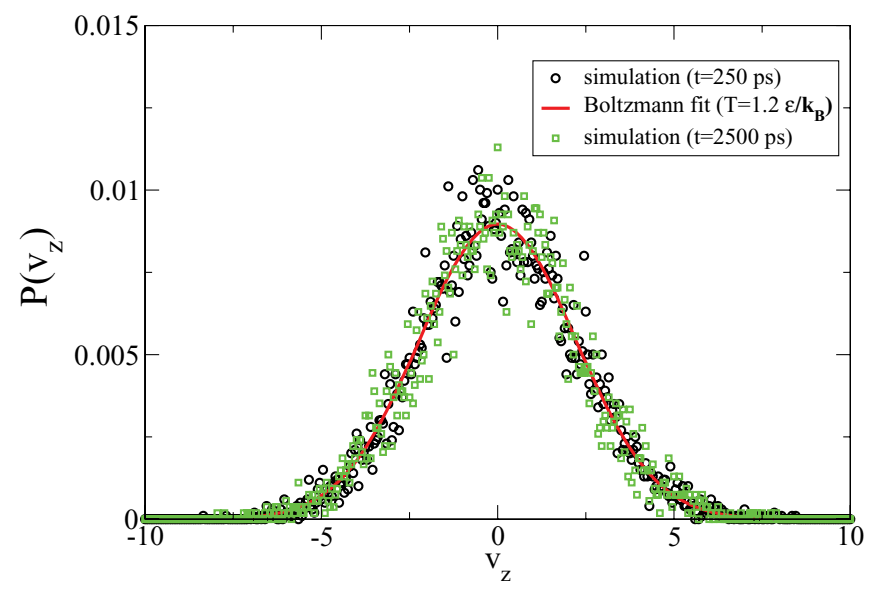

FIG. 3. (Color online) The velocity distribution function obtained from numerical simulations at $T=1.2 \epsilon / k_{\mathrm{B}}$, averaged over 9000 runs for two different times ( $t=250 \mathrm{ps}$ and $t=2500 \mathrm{ps}$ ). The simulations all collapse on a single Maxwell-Boltzmann distribution curve plotted with the solid (red) line.

boundary conditions and lies in the $z=0$ plane. All atoms in the membrane are frozen and interact with the translocating chain via the Lennard-Jones potential. Before the translocation was started, we put the middle bead of the chain symmetrically inside the pore and equilibrated the configuration, while keeping the middle bead fixed. To verify that the chain has completely relaxed to equilibrium, we wait until the average radius of gyration no longer changes with time. We have checked a posteriori that the number of translocation events to the trans- and cis-sides are evenly distributed.

The translocation process of the polymer chain is studied by Brownian dynamics simulations in a fictitious solvent. The translocation velocity $v(t)$ is sometimes approximated by $v_{z}(t)$, the $z$ component of the velocity of the bead inside the pore. From the recorded data of the translocation coordinate and the velocity $v_{z}(t)$, we make histograms for the velocityand $s$-coordinate distributions; see also Figs. 3 and 4(a). It is convenient to define the translocation coordinate $s(t)$ as a continuous variable so that one may easily calculate the translocation velocity $v(t)=d s(t) / d t$. In Fig. 2, we show a simple way to do this. The origin of the coordinate system is located in the pore. The $z$ coordinates of the $n$th and $(n+1)$ th beads are marked as $z_{n}$ and $z_{n+1}$, respectively, so that $z_{n} \geqslant 0$ and $z_{n+1} \leqslant 0$. The continuous translocation coordinate that interpolates between two consecutive integer values $n$ and $n+1$ can be defined as

$$
s=\left\{\begin{array}{lll}
n+\frac{z_{n}}{\left|z_{n+1}\right|+z_{n}} & \text { if } & z_{n} \neq 0 \text { and } z_{n+1} \neq 0 \\
n & \text { if } & z_{n}=0 \\
n+1 & \text { if } & z_{n+1}=0
\end{array}\right.
$$

We plot the the velocity distribution function $P\left(v_{z}\right)$ [see Fig. 3 and the translocation coordinate distribution $W(s, t)$ in Fig. 4(a)] for several times $t=1.0 \times 10^{4}, t=5.0 \times 10^{4}$, $t=1.0 \times 10^{5}, t=1.5 \times 10^{5}$, and $t=5.0 \times 10^{5}$ time steps. All distributions were obtained by averaging over at least 5000 runs. Note that one time step corresponds to 0.005 ps $=0.005 \sigma \sqrt{m / \epsilon}$. The velocity distribution is clearly seen from Fig. 3 to be a Gaussian, centered around $v_{z}=0$. We also plotted $P\left(v_{z}\right)$ for $t=50000$ and $t=500000$ time steps so as to demonstrate that the distribution is indeed time independent. Moreover, we verified that the distribution is well described by a Maxwell-Boltzmann distribution for the simulation temperature $T=1.2 \epsilon / k_{\mathrm{B}}$. The PDF for the $s$ coordinate $W(s, t)$ is centered around $s=51$, which is the middle bead of the chain of length $N=101$; the distribution broadens symmetrically. From Figs. 3 and 4(a), it can easily be seen that the distributions for both the velocity and the translocation coordinate $s$, obtained from our molecular dynamics simulation, are indeed Gaussian. In Fig. 4(b), we show the change of the variance $\Delta(t)$ [as defined by Eq. (14)] of the Gaussian distribution curves $W(s, t)$ as a function of time.

Evidently, $\Delta(t)$ behaves subdiffusively as $\Delta \propto t^{0.55}$ for shorter times, and later switches to a $t^{0.91}$ law for longer times. This corresponds to the simple scaling consideration [30],
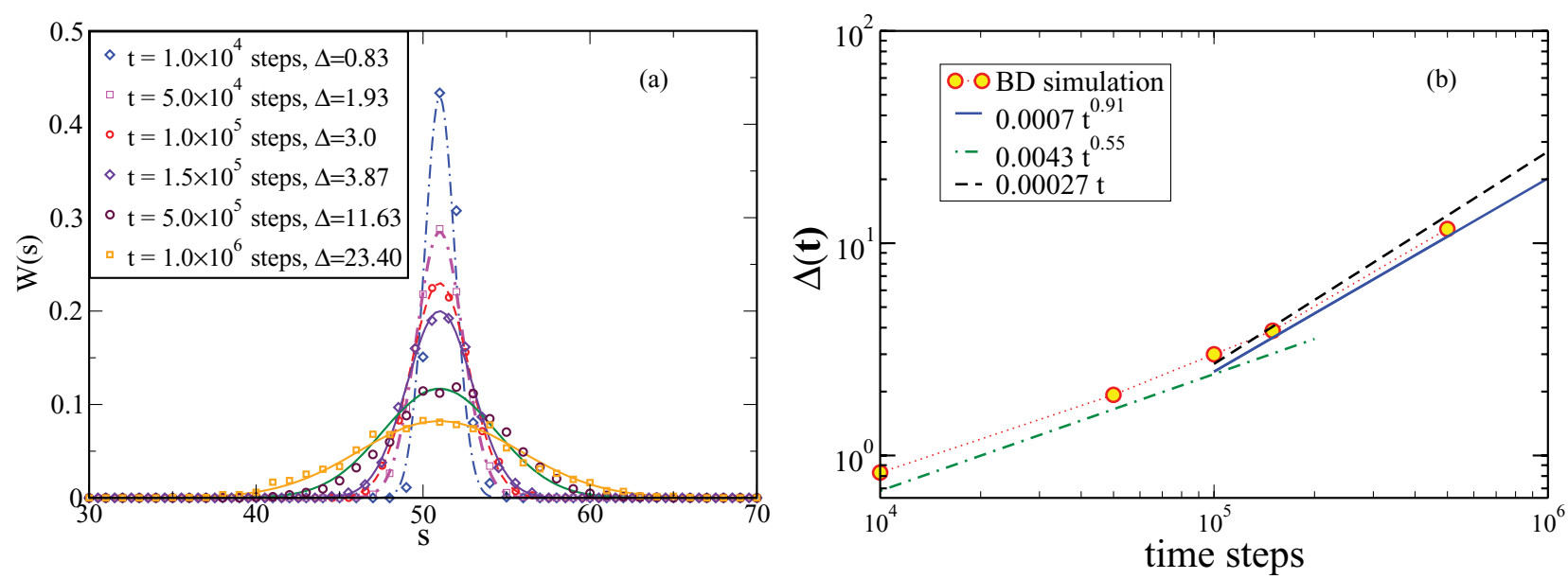

FIG. 4. (Color online) (a) The PDF of the translocation coordinate $W(s, t)$ at five different time moments. The five Gaussian fits correspond to the PDF of $s$ at time steps $10^{4}$ to $10^{6}$ and have a variance $\Delta(t)$, the value of which is given in the legend and that increases with the number of time steps. (b) The variance $\Delta(t)$ of the distribution $W(s, t)$ as a function of number of time steps. It is clear that the behavior for short times is subdiffusive and scales as $t^{0.55}$. For larger times $t>10^{5}$ time steps, the behavior changes and is only slightly subdiffusive and $\Delta(t) \simeq t^{0.91}$. 

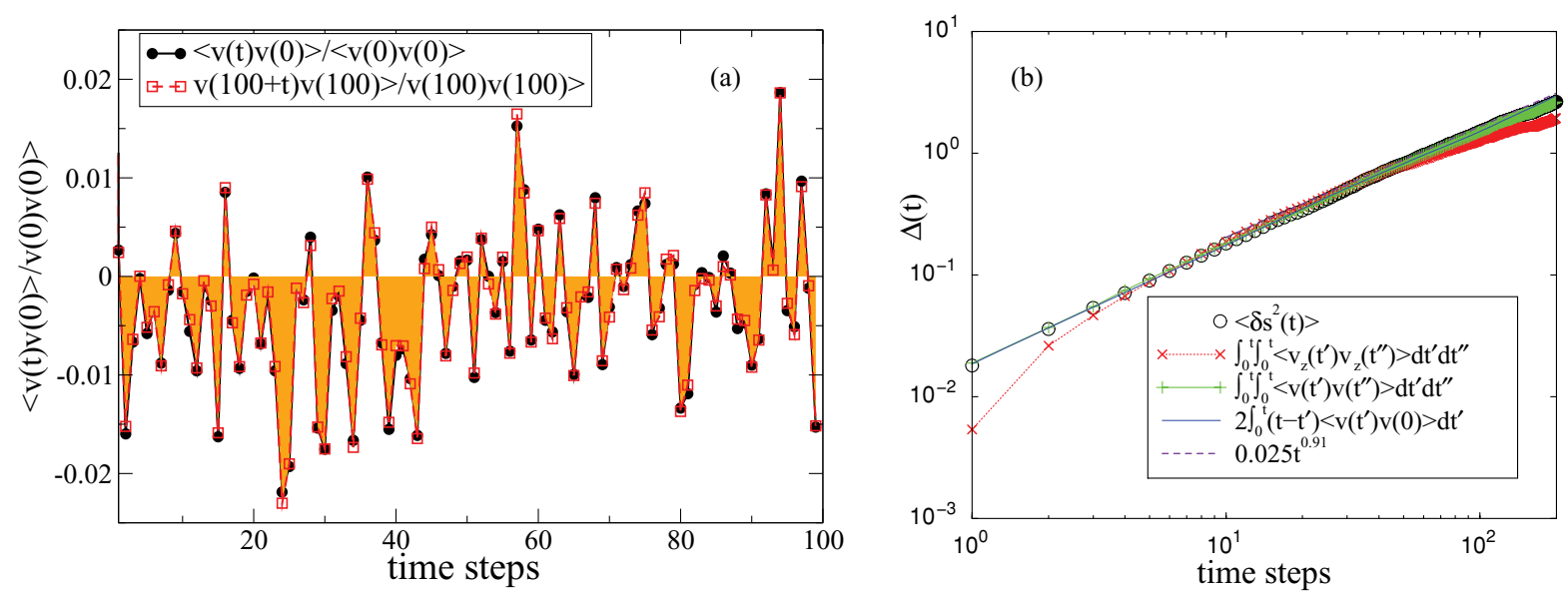

FIG. 5. (Color online) (a) The normalized velocity correlator against elapsed time $t$. (b) Comparison of the $\Delta(t)$ data (circles) with the result of integration of the velocity correlator according to Eq. (2) (solid line). The double time integral $\int_{0}^{t} d t^{\prime} \int_{0}^{t^{\prime}} d t^{\prime \prime}\left\langle v\left(t^{\prime}\right) v\left(t^{\prime \prime}\right)\right\rangle$, which is valid even when time-translational invariance does not hold, is given by (green) + symbols.

which claims that the variance of the translocation coordinate $\Delta(t) \equiv\left\langle s^{2}\right\rangle-\langle s\rangle^{2}$ in the long-time limit should go as $\Delta(t) \propto$ $t^{\alpha}$, where $\alpha=2 /(2 v+1) \approx 0.92$ for the three-dimensional case. This power is obtained by the requirement that the translocation time $\tau_{\mathrm{tr}}$ should scale like the maximal relaxation time of the polymer chain, i.e., $\tau_{\mathrm{tr}} \propto N^{2 v+1}$. In addition, by the time the translocation is completed, the following relationship holds: $\Delta\left(\tau_{\text {tr }}\right) \propto N^{2}$.

We emphasize that the change in the exponent $\alpha$ from 0.55 in the range $\left(10^{4}, 10^{5}\right)$ time steps to 0.91 from $\left(10^{5}, 10^{6}\right)$ time steps is in agreement with recently reported results by Amatai et al. [14], who considered the movement of a tagged monomer in a Rouse chain. This somewhat surprising result indicates that the study of the movement of a tagged Rouse monomer can indeed provide essential information about the translocation process.

Next,we consider the velocity correlation functions. The normalized auto-correlation function is depicted in Fig. 5(a). From this figure, one can infer that the correlation time is not zero, although it is rather small. One clearly sees anticorrelation in the interval $t \in(0,5)$, after which the correlations vanish on the average. Note that such unusual negative correlations at short-time lags have been observed recently in experimental studies of the subdiffusive motion of bacterial chromosomal loci through a viscoelastic cytoplasm [31]. To illustrate the invariance with respect to shifts in time, a time-shifted correlation function is also displayed. Since the curves collapse onto each other, this demonstrates that the correlation function is indeed time invariant as required. In Fig. 5(b), we give the consistency check: the translocation coordinate mean-square displacement $\Delta(t)$ as derived from the MD simulation is compared to the time integral given by Eq. (2). One can see very good agreement between these two ways of $\Delta(t)$ calculation, which also suggests that the velocity correlator [cf. Fig. 5(a)] has been found with reasonable accuracy.

We have also relaxed the time-translational invariance assumption and represented $\Delta(t)$ as a double integral $\int_{0}^{t} d t^{\prime} \int_{0}^{t^{\prime}} d t^{\prime \prime}\left\langle v\left(t^{\prime}\right) v\left(t^{\prime \prime}\right)\right\rangle$. The results of these calculations basically superimpose onto each other, which indicates again that the time-translational invariance indeed holds. From
Fig. 5, it can also be seen that the $z$ component of the monomer velocity inside the pore provides a reasonable measure for the translocation velocity, although there definitely are minor deviations between this velocity and the translocation velocity. In particular, the variance of the tagged particle velocity has a somewhat smaller power-law exponent than the real translocation velocity. However, combining Fig. 5(b) with Fig. 4(b) shows that the exponent $\alpha$ changes with time from 0.91 on the very-short-time scales (1200) to about 0.55 for times on the order of $\times 10^{4}$. This is again in agreement with the prediction of [14] for a tagged Rouse monomer.

Figure 6 demonstrates the $\Delta(t)$ behavior for a larger time interval where it is seen to reach a plateau as soon as the polymer chain has completed the translocation process. Here, we compare the variance $\Delta(t)$ from numerical simulation with the analytical expression equation (14), where we took $\alpha=0.92$. It can be clearly seen that the results are in good agreement.

The first-passage-time distribution $Q(\tau)$ is interesting too. In a previous paper [5], we employed the FFPE to describe the



FIG. 6. (Color online) $\langle\Delta(t)\rangle$ for long times reaches a plateau. The plateau height as well as the slope of the theoretical curve preceding the plateau are in a good agreement with simulation results. 


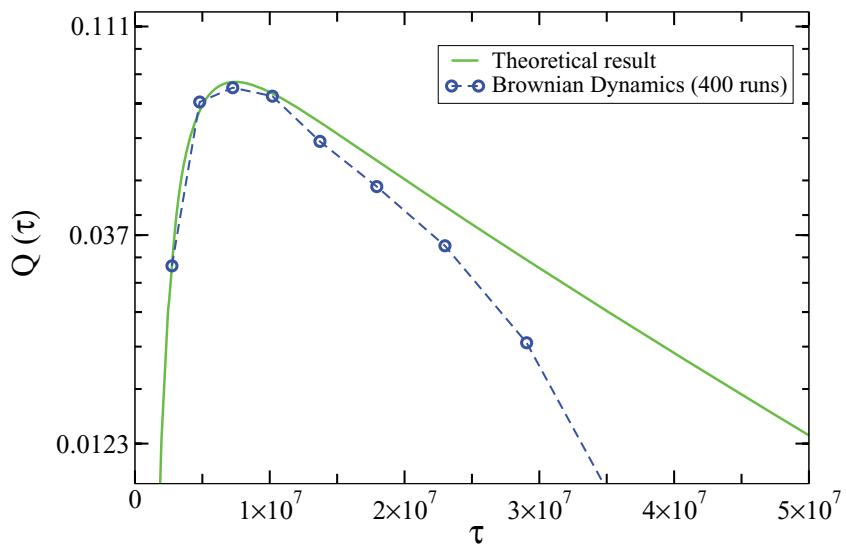

FIG. 7. (Color online) The FPT distribution $Q(\tau)$. Theory and simulations compared for an $N=51$ chain.

distribution $Q(\tau)$. The tail of the distribution decreased as a power law in that case. However, for the theory that we develop here, a stretched-exponential decay is expected. For an $N=51$ chain, we have performed simulations to compare $Q(\tau)$ with the theoretical prediction. For an average over 400 runs, we obtained the result that is shown in Fig. 7. The theoretical distribution is found to be in reasonable agreement with the simulation result. We remark that experimental and numerical verification of the tail of the translocation time distribution is extremely difficult, as the events constituting the distribution's tail are very sparse.

\section{CONCLUSION}

In this work, we treat the translocation dynamics within the framework of fractional Brownian motion whereby the definition of the translocation coordinate velocity $d s(t) / d t=$ $v(t)$ is considered as a Langevin equation, where $v(t)$ can be viewed as a Gaussian process with the two first cumulants given by: $\langle v(t)\rangle=f(t) / \xi_{0}$ and $G(t)=\langle v(t) v(0)\rangle-\langle v(t)\rangle^{2}$. The first cumulant implies that the friction $\xi_{0}$ is constant, in agreement with data from computer experiments. This does not rule out the possibility that there are cases in which friction might be time dependent [10]. In such cases, a modified version of the governing equation (7) would be warranted.

Based on this Langevin equation, we derive (without any further assumption) the appropriate Fokker-Planck equation of motion for the distribution function $W(s, t)$ with a timedependent drift and diffusion coefficient. The obtained solution for $W(s, t)$ demonstrates two characteristic features of $\mathrm{fBm}$, which agree favorably with the recent findings [12-14] as regards nondriven translocation dynamics: (i) A Gaussian distribution for the translocation coordinate $s$ during the translocation process and (ii) a subdiffusive behavior for the variance of the translocation coordinate $\Delta(t)=\left\langle s^{2}(t)\right\rangle-$ $\langle s(t)\rangle^{2}$. Moreover, the survival probability $S\left(t, s_{0}\right)$ in the long-time limit has a stretched-exponential form in agreement with recent findings [29] (albeit in contrast to a popular opinion about its scaling behavior [28]). One should note, however, that the power $(0.92)$ of the stretched-exponential function is very close to unity so that a distinction from simple exponential decay of the survival probability at the long-time limit would be hardly detectable.

The velocity correlator $G(t)$ for the case of unbiased translocation has been computed by means of Brownian dynamics simulation and found to expose unusual negative correlations in time. A consistency check, implemented by means of Eq. (2), shows that the calculated $G(t)$ is meaningful indeed. Thus, it appears that the presence of negative velocity correlations reveals a feature that is specific for the process of translocation in particular and for anomalous diffusion in general [31].

We have calculated the variance of the translocation coordinate $\Delta(t)$ and shown that $\Delta(t)$ follows a power law, with different exponents $\alpha$, depending on the time scales in which one is interested. In particular, for times on the order of the translocation time, $\Delta \sim t^{0.91}$ in 3D. Furthermore, we examined the translocation-time distribution function $Q(\tau)$ derived from simulation results in a broad time interval and compared these to our theoretical predictions. Unfortunately, attaining good statistics for very late times still remains a difficult task for present-day simulation studies, so more work will be needed in order to test the agreement with theoretical predictions unambiguously.

We have shown in Sec. II that our approach can be used for the biased translocation as well. This investigation will be reported on in a separate presentation.

\section{ACKNOWLEDGMENTS}

We are thankful to our late colleague and collaborator S. Kotsev who took part in the beginning of this investigation. The authors are indebted to A. Y. Grosberg for stimulating discussions. We gratefully acknowledge Project No. SFB-DFG 625 for financial support. A. M. appreciated the hospitality during his stay at the Max-Planck Institute for Polymer Research in Mainz. J. L. A. D. is grateful for the technical support of P. Theodorakis with the GROMACS computations.
[1] A. Meller, J. Phys. Condens. Matter 15, R581 (2003).

[2] M. Zwolak and M. Di Ventra, Rev. Mod. Phys. 80, 141 (2008).

[3] K. Luo, T. Ala Nissila, and S.-Ch. Ying, J. Chem. Phys. 124, 034714 (2006).

[4] I. Huopaniemi, K. Luo, T. Ala-Nissila, and S.-Ch. Ying, J. Chem. Phys. 125, 124901 (2006).

[5] J. L. A. Dubbeldam, A. Milchev, V. G. Rostiashvili, and T. A. Vilgis, Phys. Rev. E 76, 010801(R) (2007).
[6] J. L. A. Dubbeldam, A. Milchev, V. G. Rostiashvili, and T. A. Vilgis, Europhys. Lett. 79, 18002 (2007).

[7] D. Panja, G. T. Barkema, and R. C. Ball, J. Phys. Condens. Matter 19, 432202 (2007).

[8] H. Vocks, D. Panja, G. T. Barkema, and R. C. Ball, J. Phys. Condens. Matter 20, 095224 (2008).

[9] D. Panja and G. T. Barkema, Biophys. J. 94, 1630 (2008); 94, 095224 (2008).

[10] T. Sakaue, Phys. Rev. E 76, 021803 (2007); 81, 041808 (2010). 
[11] R. Metzler and J. Klafter, Biophys. J. 85, 2776 (2003).

[12] Y. Kantor and M. Kardar, Phys. Rev. E 76, 061121 (2007).

[13] C. Chatelain, Y. Kantor, and M. Kardar, Phys. Rev. E 78, 021129 (2008).

[14] A. Amitai, Y. Kantor, and M. Kardar, Phys. Rev. E 81, 011107 (2010).

[15] B. J. West, M. Bologna, and P. Grigolini, Physics of Fractal Operators (Springer-Verlag, New York, 2003).

[16] J.-P. Boon and S. Yip, Molecular Hydrodynamics (McGraw-Hill, New York, 1980), Sec. 2.5.

[17] E. A. Novikov, Zh. Eksp. Theor. Fiz. 47, 1919 (1964) [Sov. Phys. JETP 20, 1290 (1965)].

[18] J. Zinn-Justin, Quantum Field Theory and Critical Phenomena (Oxford University Press, New York, 1993), Sec. 4.2.

[19] R. Metzler and J. Klafter, Phys. Rep. 339, 1 (2000).

[20] A. Bhattacharya, W. H. Morrison, K. Luo, T. Alla-Nissila, S.-C. Ying, A. Milchev, and K. Binder, Eur. Phys. J. E 29, 423 (2009).
[21] S. Chaudhury and B. J. Cherayil, J. Chem. Phys. 125, 024904 (2006).

[22] S. Chaudhury and B. J. Cherayil, J. Phys. Chem. 112, 15973 (2008).

[23] D. Panja, e-print arXiv:1007.0378.

[24] D. Panja, J. Stat. Mech. (2010) L02001; (2010) P06011.

[25] R. Mannella, P. Grigolini, and B. J. West, Fractals 2, 81 (1994).

[26] P. Allegrini, M. Buiatti, P. Grigolini, and B. J. West, Phys. Rev. E 57, 4558 (1998).

[27] H. Risken, The Fokker-Planck Equation (Springer-Verlag, Berlin, 1989).

[28] A. Zoia, A. Rosso, and S. N. Majumdar, Phys. Rev. Lett. 102, 120602 (2009).

[29] G. Oshanin, e-print arXiv:0801.0676; e-print arXiv:0801.2914.

[30] J. Chuang, Y. Kantor, and M. Kardar, Phys. Rev. E 65, 011802 (2001).

[31] S. C. Weber, A. J. Spakowitz, and J. A. Theriot, Phys. Rev. Lett. 104, 238102 (2010). 